\title{
Anatomical features of the superficial temporal artery in hemorrhagic moyamoya disease based on CT angiography
}

\author{
KUN HOU* ${ }^{*}$, QINGLIAN LI* ${ }^{*}$ KAN XU, BAOFENG XU And JINLU YU \\ Department of Neurosurgery, The First Hospital of Jilin University, Changchun, Jilin 130021, P.R. China
}

Received May 9, 2019; Accepted December 11, 2019

DOI: $10.3892 /$ etm.2020.8473

\begin{abstract}
The superficial temporal artery (STA) is an important continuation of the external carotid artery. It is divided into the frontal branch and the parietal branch at or above the zygomatic arch. In the present study, a comparative analysis of the STA in patients with and without moyamoya disease (MMD) was performed using CT angiography. Patients admitted to our institution for spontaneous intracranial hemorrhage were potential candidates. In general, 25 cases (50 sides) in the MMD group and 25 cases (50 sides) in the non-MMD group were selected for evaluation. The morphology of the STA when crossing the zygomatic arch, the association between the STA bifurcation and the zygomatic arch, the branching characteristics of the STA, the parameters of the STA bifurcation point, the diameter of the STA and the distance from the origin of the STA to the bifurcation point were selected for analysis. There were no significant differences between the two groups with regard to the association between the STA bifurcation and the zygomatic arch, the diameter of the STA or the distance from the origin of the STA to the bifurcation point. However, the bifurcation point of the STA was closer to the posterior edge of the mandibular condyle in the patients with MMD.
\end{abstract}

\section{Introduction}

The superficial temporal artery (STA) is an important continuation of the external carotid artery. It is divided into the frontal branch and the parietal branch at or above the zygomatic arch (1). In neurosurgical practice, the STA is an

Correspondence to: Dr Jinlu Yu, Department of Neurosurgery, The First Hospital of Jilin University, 1 Xinmin Avenue, Changchun, Jilin 130021, P.R. China

E-mail: jlyu@jlu.edu.cn

*Contributed equally

Abbreviations: CTA, CT angiography; ICH, intracranial hemorrhage; MMD, moyamoya disease; STA, superficial temporal artery

Key words: moyamoya disease, superficial temporal artery, intracranial hemorrhage, CT angiography excellent donor vessel for extracranial-intracranial bypass (2). In previous studies, the diameter of the STA, the association between its bifurcation point and the zygomatic arch, its characteristics when crossing the zygomatic arch and its classifications were the parameters assessed. Most of the studies were performed using cadaveric measurements in the general population and had small sample sizes $(3,4)$.

Certain studies have evaluated the anatomical characteristics of the STA in patients with moyamoya disease (MMD). MMD is an idiopathic chronic occlusive cerebrovascular disease characterized by stenosis of the terminal internal carotid artery (ICA) and/or the proximal portion of the anterior cerebral artery and/or the middle cerebral artery, as well as development of basal moyamoya vessels (5). At present, extracranial-intracranial bypass is an effective method for the treatment of MMD and the STA is the major source of donor vessels (6). However, whether the STA is also affected in patients with MMD has remained elusive. In the present study, a comparative analysis of the STA in patients with and without MMD was performed using CT angiography (CTA).

\section{Materials and methods}

Patients. Patients admitted to our institution for spontaneous intracranial hemorrhage (ICH) between January 2017 and January 2018 were considered as potential candidates. All patients underwent head CTA. If the CTA indicated steno-occlusive changes in the ICA terminus and/or the beginning of its two major branches, further digital subtraction angiography was performed for the definite diagnosis of MMD. Patients who had an underlying disease that may cause steno-occlusive changes in the ICA terminus (moyamoya syndrome) were excluded. According to the CTA and DSA results, ICH cases were divided into MMD and non-MMD groups. The patients included were required to have discernible STAs and/or STA branches on CTA.

Workstation and indexes measured. Raw-image CTA data were reconstructed in the Volume Rendering program of the GE Workstation (version 4.6; GE Healthcare) to observe the anatomical characteristics of the STA.

Morphology of the STA when crossing the zygomatic arch. According to their morphology when crossing the zygomatic 
arch, the STAs were divided into the tortuous (T-type; Fig. 1A) and straight type (S-type; Fig. 1B).

Association between the STA bifurcation and the zygomatic arch. According to the vertical association between the STA bifurcation and the zygomatic arch, the STAs were divided into three types: i) Bifurcation above the upper edge of the zygomatic arch (Fig. 2A), ii) bifurcation at the zygomatic arch (Fig. 2B) and iii) bifurcation below the lower edge of the zygomatic arch (Fig. 2C).

Branching characteristics of the STA. The STAs were subdivided into the following types based on their branching characteristics: Type A: STA with equal bifurcation (Fig. 3A); type B: Atrophic frontal branch (Fig. 3B); type C: Atrophic parietal branch (Fig. 3C); type D: Additional frontal branch (Fig. 3D); type E: Additional parietal branch (Fig. 3E); type F: Parietal branch only (Fig. 3F); type G: Frontal branch only (Fig. 3G).

Parameters of the STA bifurcation point. For patients with an STA bifurcation point above the zygomatic arch, 3 parameters were used, as illustrated in Fig. 4. Line a was defined as the distance from the bifurcation point to the posterior edge of the mandibular condyle. Line a is parallel to the long axis of the zygomatic arch. Line $b$ was defined as the vertical distance from the STA bifurcation point to the upper edge of the zygomatic arch. Line c was defined as the distance from the STA bifurcation point to the zygomatic process of the frontal bone. Line $\mathrm{c}$ is also parallel to the long axis of the zygomatic arch (Fig. 4).

Diameter of the STA. Cases with bifurcation points below the zygomatic arch and those whose STA did not bifurcate were excluded from this measurement. The diameter of the STA was measured at the beginning, the midpoint of the zygomatic arch and the bifurcation point.

Distance from the origin of the STA to the bifurcation point. Patients whose STA did not bifurcate were excluded from this measurement. The Two-Click AVA tool was used to select two points at the beginning and the bifurcation of the STA, and the workstation then automatically measured the length.

Statistical analysis. SPSS 18.0 (IBM Corp.) was used for statistical analysis. The normal distribution for continuous variables was assessed using a Shapiro-wilk test. Continuous variables are expressed as the mean \pm standard deviation and differences were assessed with Student's t-test. Categorical variables were described as the number of cases (percentage) and were assessed with the $\chi^{2}$ test or Fisher's exact test. $\mathrm{P}<0.05$ was considered to indicate statistical significance.

\section{Results}

General information. A total of 25 consecutive cases (50 sides) were finally selected as the MMD group and 25 control cases as the non-MMD group. In the MMD group, the average age
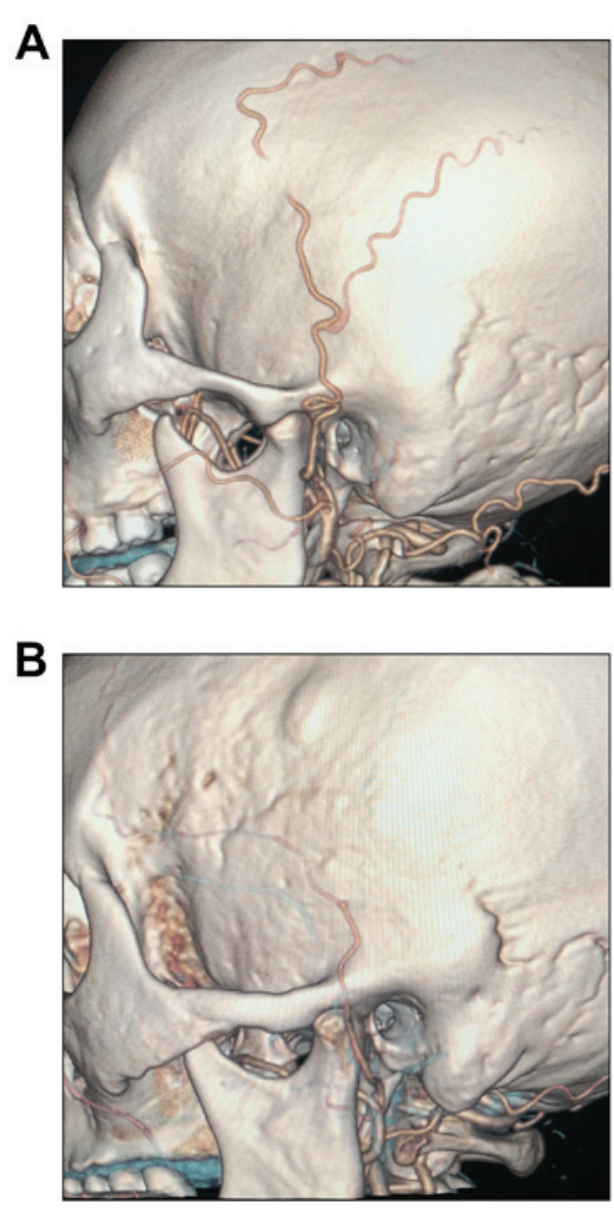

Figure 1. Representative CT angiography images for different types of STA (A) The STA is tortuous when crossing the zygomatic arch (T-type). (B) The STA is straight when crossing the zygomatic arch. STA, superficial temporal artery (S-type).

of the patients was 54.6 \pm 9.3 years (range, 27-71 years) and the ratio of males to females was 1.1:1. In the non-MMD group, the average age was $55.8 \pm 11.3$ years (range, $30-77$ years) and the ratio of males to females was 2.1:1. There was no statistical difference $(\mathrm{P}=0.248)$ in gender ratio between the MMD and non-MMD groups.

Morphology of the STA when crossing the zygomatic arch. In the MMD group, 44 sides (88\%) were type T and 6 sides (12\%) were type S. In the non-MMD group, 45 sides (90\%) were type $\mathrm{T}$ and 5 sides (10\%) were type $\mathrm{S}$. No significant difference was present between the two groups in terms of the distribution of T- and S-types ( $\mathrm{P}=0.749$; Table I).

Association between the STA bifurcation point and the zygomatic arch. In the MMD group, 8 sides without STA bifurcation were identified and excluded. In the remaining sides, the bifurcation points were located above the upper edge of the zygomatic arch on 37 sides (88.1\%) and directly on the zygomatic arch on 5 sides (11.9\%). None of the patients had an STA that bifurcated below the lower edge of the zygomatic arch. In the non-MMD group, 8 sides with no bifurcation were excluded. In the remaining sides, the bifurcation points were located above the upper edge of the zygomatic arch on 37 sides $(88.1 \%)$, directly on the zygomatic arch on 4 sides 

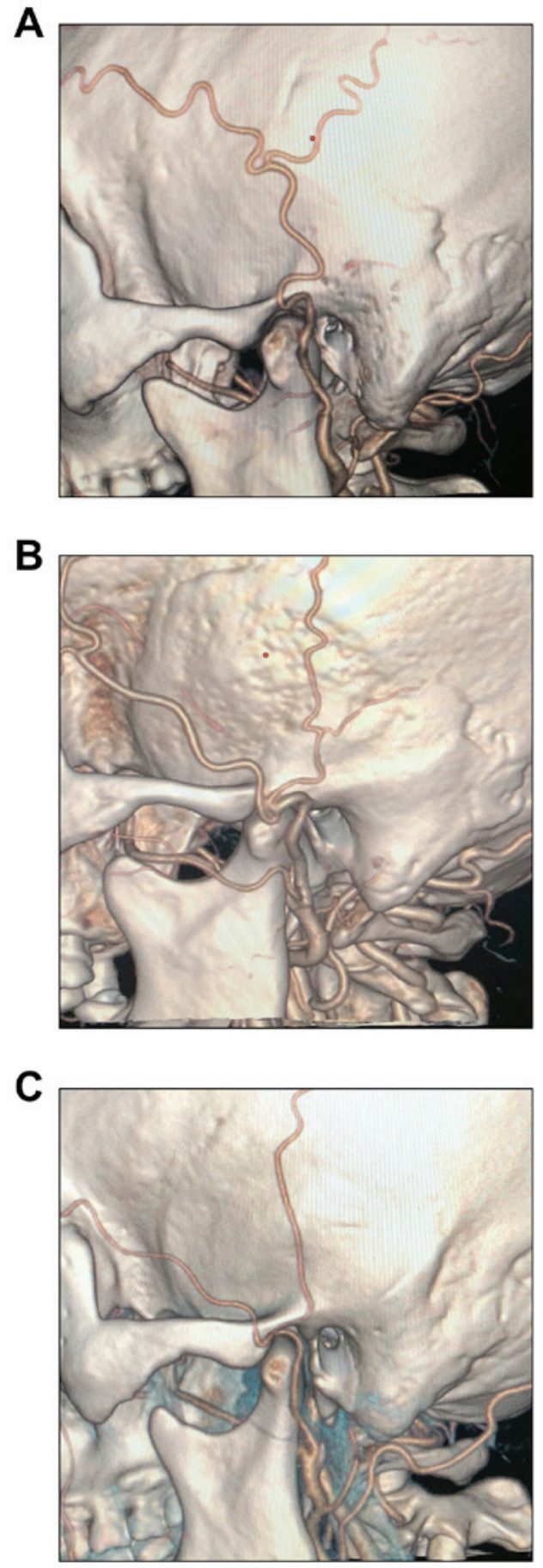

Figure 2. Representative CT angiography images for different locations of the STA bifurcation point relative to the zygomatic arch. (A) The bifurcation point of the STA is above the upper edge of the zygomatic arch. (B) The bifurcation point is at the zygomatic arch. (C) The bifurcation is below the lower edge of the zygomatic arch. STA, superficial temporal artery.

(9.5\%) and below the lower edge of the zygomatic arch on 1 side $(2.4 \%)$. No significant difference was present between the two groups with regard to the distribution of different associations between the STA bifurcation and the zygomatic $\operatorname{arch}(\mathrm{P}=1.000$; Table II).

Branching characteristics of the STA. In the MMD group, the branching characteristic was type A, B, C, D, E, F and G on 25 sides (50\%), 8 sides (16\%), 6 sides (12\%), 3 sides (6\%), 0
A
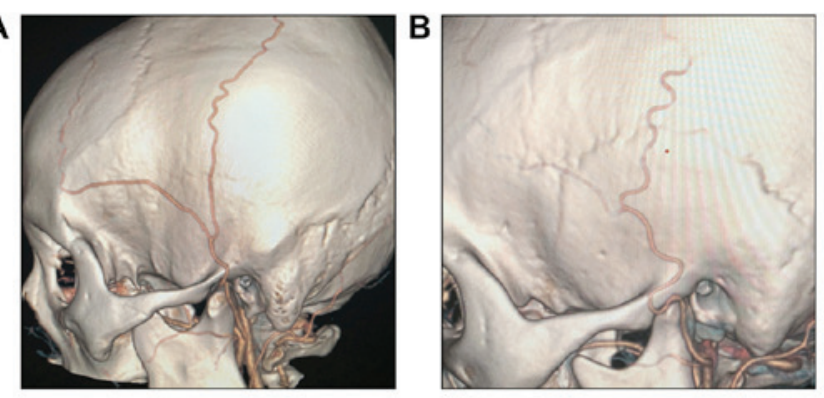

C
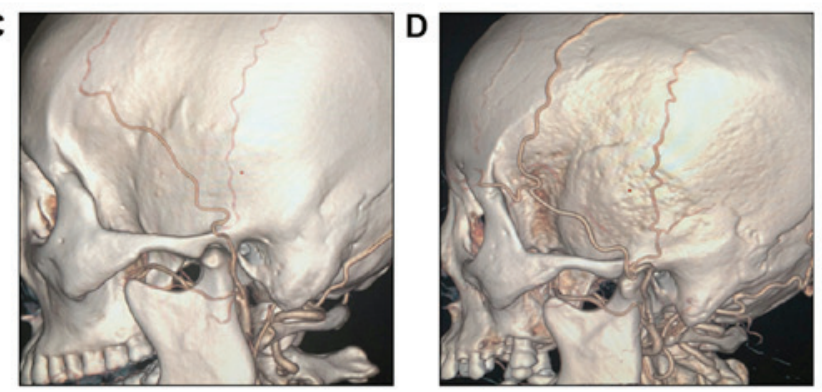

E
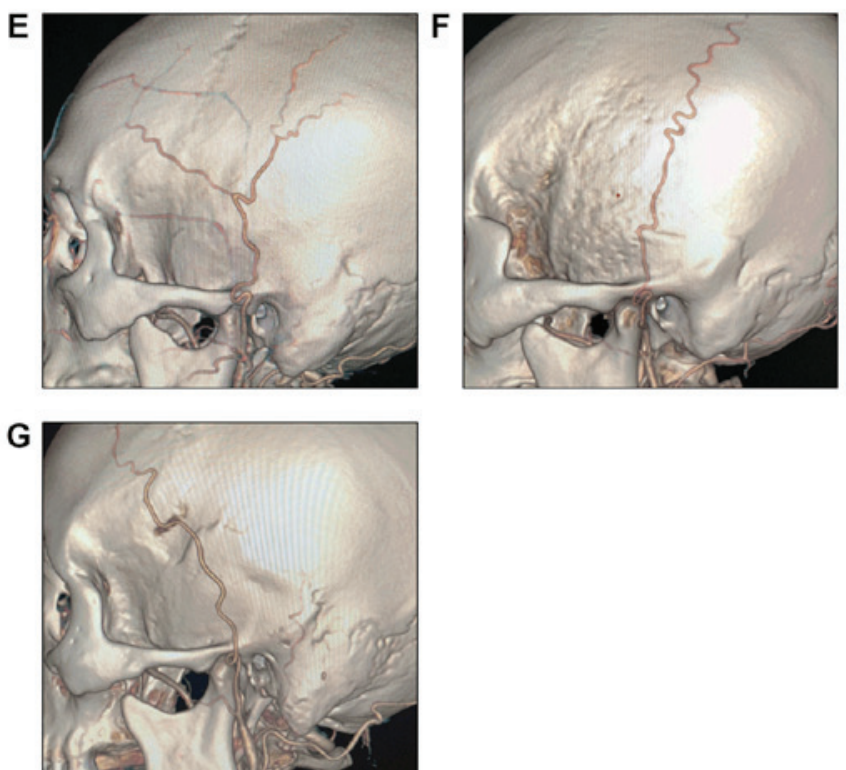

Figure 3. Representative CT angiography images for different branching types of the STA. (A) Type A: The frontal and parietal branches of the STA are equal at the bifurcation; (B) type B: The frontal branch is atrophic; (C) type C: The parietal branch is atrophic; (D) type D: The frontal branch bifurcates further; (E) type E: The parietal branch bifurcates further; (F) type F: The STA continues as the parietal branch only; $(\mathrm{G})$ type G: The STA continues as the frontal branch only. STA, superficial temporal artery.

side $(0 \%), 4$ sides $(8 \%)$ and 4 sides $(8 \%)$, respectively. In the non-MMD group, the branching characteristic was type A, B, C, D, E, F and G in 19 sides (38\%), 3 sides (6\%), 5 sides (10\%), 8 sides (16\%), 7 sides (14\%), 4 sides (8\%) and 4 sides (8\%), respectively. Overall, a statistical difference was indicated in the component ratios of each branching type between the two groups $(\mathrm{P}=0.042)$. Regarding the individual types, only the incidence of type $\mathrm{E}$ was significantly different between the two groups ( $\mathrm{P}=0.012$; Table III).

Comparison of measurements of the STA bifurcation point. The STA bifurcation point was above the zygomatic arch on 37 sides in the MMD group and 38 sides in the non-MMD 
Table I. Distribution of patients with/without MMD regarding the morphology of the superficial temporal artery when crossing the zygomatic arch.

\begin{tabular}{lccr}
\hline Zygomatic arch crossing type $^{\mathrm{a}}$ & MMD (n=50) & Non-MMD (n=50) & P-value \\
\hline $\mathrm{S}$ & $6(12)$ & $5(10)$ & 0.749 \\
$\mathrm{~T}$ & $44(88)$ & $45(90)$ & \\
\hline
\end{tabular}

aZygomatic arch crossing type $\mathrm{T}$ and $\mathrm{S}$ as indicated in Fig. 1A and B, respectively. Values are expressed as n (\%). MMD, moyamoya disease; $\mathrm{S}$, straight type; $\mathrm{T}$, tortuous type.

Table II. Distribution of patients with/without MMD regarding the location of the STA bifurcation point relative to the zygomatic arch.

\begin{tabular}{lcccr}
\hline Location of STA bifurcation point $^{\mathrm{a}}$ & Total (n=84) & MMD (n=42) & Non-MMD (n=42) & P-value \\
\hline Above the upper edge of the zygomatic arch & $74(88.1)$ & $37(88.1)$ & $37(88.1)$ & 1.000 \\
At the zygomatic arch & $9(10.7)$ & $5(11.9)$ & $4(9.5)$ & $1(2.4)$ \\
Below the lower edge of the zygomatic arch & $1(1.2)$ & $0(0.0)$ & \\
\hline
\end{tabular}

${ }^{\mathrm{a}}$ As illustrated in Fig. 2. Values are expressed as n (\%). MMD, moyamoya disease; STA, superficial temporal artery.

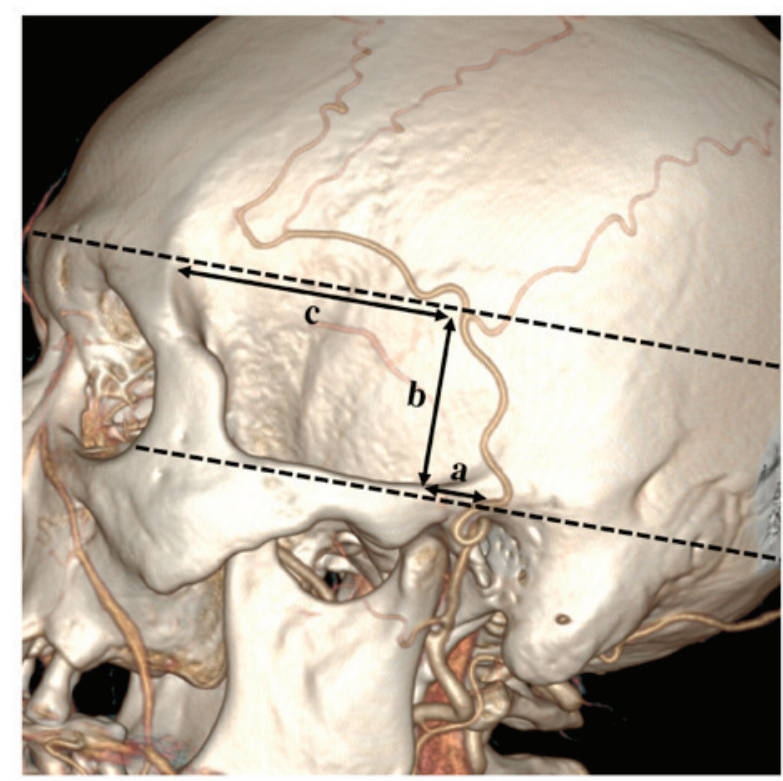

Figure 4. Line a is parallel to the long axis of the zygomatic arch. It denotes the distance from the bifurcation point to the posterior edge of the mandibular condyle. Line $b$ is the vertical distance from the STA bifurcation point to the upper edge of the zygomatic arch. Line $\mathrm{c}$ is the distance from the STA bifurcation point to the zygomatic process of the frontal bone. Line $\mathrm{c}$ is in parallel with line a. STA, superficial temporal artery.

group. The mean values of lines a, b and c were $13.35 \pm 4.07$, $27.16 \pm 9.83$ and $54.71 \pm 3.71 \mathrm{~mm}$ in the MMD group, respectively. The mean values of lines a, $\mathrm{b}$ and $\mathrm{c}$ were $15.43 \pm 4.63$, $29.60 \pm 8.96$ and $55.18 \pm 6.73 \mathrm{~mm}$ in the non-MMD group, respectively. The values of lines $\mathrm{a}, \mathrm{b}$ and $\mathrm{c}$ were normally distributed and Student's t-test was used for the intergroup comparison. In the MMD group, line a was significantly larger
Table III. Branching characteristics of the superficial temporal artery.

\begin{tabular}{lccc}
\hline Branching type $^{\mathrm{a}}$ & $\begin{array}{c}\text { MMD } \\
(\mathrm{n}=50)\end{array}$ & $\begin{array}{c}\text { Non-MMD } \\
(\mathrm{n}=50)\end{array}$ & P-value \\
\hline Total & & & 0.042 \\
A & $25(50)$ & $19(38)$ & 0.314 \\
B & $8(16)$ & $3(6)$ & 0.200 \\
C & $6(12)$ & $5(10)$ & 1.000 \\
D & $3(6)$ & $8(16)$ & 0.200 \\
E & $0(0)$ & $7(14)$ & 0.012 \\
F & $4(8)$ & $4(8)$ & 1.000 \\
G & $4(8)$ & $4(8)$ & 1.000 \\
\hline
\end{tabular}

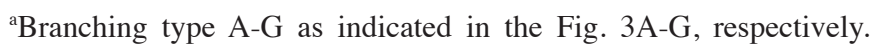
Values are expressed as n (\%). MMD, moyamoya disease.

than that in the non-MMD group $(\mathrm{P}=0.045)$. No significant difference was noted between the two groups with regard to lines $b$ and $c$ (Table IV).

Diameter of the STA. In the MMD group, the diameters at the beginning (50 sides), the midpoint of the zygomatic arch (50 sides) and the bifurcation (42 sides) were $2.45 \pm 0.46$, $1.68 \pm 0.44$ and $1.95 \pm 0.39 \mathrm{~mm}$, respectively. In the non-MMD group, the diameters at the beginning (50 sides), the zygomatic arch midpoint (50 sides), and the bifurcation (42 sides) were $2.41 \pm 0.58,1.71 \pm 0.51$ and $1.87 \pm 0.57 \mathrm{~mm}$, respectively. The values of the diameters at each point were all normally distributed. Student's t-test indicated no significant differences with regard to the diameters of the STA at the beginning, the 
Table IV. Parameters of the superficial temporal artery bifurcation point ( $\mathrm{mm})$.

\begin{tabular}{lccr}
\hline Distance $^{\mathrm{a}}$ & MMD (n=37) & Non-MMD (n=38) & P-value \\
\hline a & $13.35 \pm 4.07$ & $15.43 \pm 4.63$ & 0.045 \\
$\mathrm{~b}$ & $27.16 \pm 9.83$ & $29.60 \pm 8.96$ & 0.268 \\
c & $54.71 \pm 3.71$ & $55.18 \pm 6.73$ & 0.713
\end{tabular}

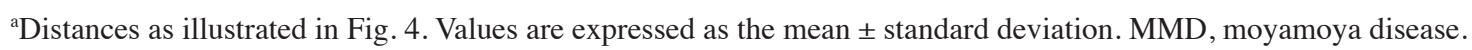

Table V. Diameter of thesuperficial temporal artery ( $\mathrm{mm})$.

\begin{tabular}{lcccrr}
\hline & \multicolumn{2}{c}{ MMD $(\mathrm{n}=50)$} & & \multicolumn{2}{c}{ Non-MMD (n=50) } \\
\cline { 2 - 3 } Location & Cases $(\mathrm{n})$ & Mean \pm SD & & Cases (n) & Mean \pm SD \\
\hline Beginning & 50 & $2.45 \pm 0.46$ & & 50 & $2.41 \pm 0.58$ \\
Midpoint of the zygomatic arch & 50 & $1.68 \pm 0.44$ & & 49 & 0.690 \\
Bifurcation & 42 & $1.95 \pm 0.39$ & 42 & 0.721 \\
\hline
\end{tabular}

MMD, moyamoya disease; SD, standard deviation.

Table VI. Distance from the origin of the superficial temporal artery to the bifurcation point (mm).

\begin{tabular}{|c|c|c|c|c|c|}
\hline \multirow[b]{2}{*}{ Item } & \multicolumn{2}{|c|}{$\operatorname{MMD}(n=50)$} & \multicolumn{2}{|c|}{ Non-MMD $(\mathrm{n}=50)$} & \multirow[b]{2}{*}{$\mathrm{P}$-value } \\
\hline & Cases (n) & Mean \pm SD & Cases (n) & Mean \pm SD & \\
\hline Distance & 42 & $73.89 \pm 15.47$ & 42 & $78.71 \pm 21.68$ & 0.244 \\
\hline
\end{tabular}

MMD, moyamoya disease; SD, standard deviation.

midpoint of the zygomatic arch and the bifurcation between the two groups (Table V).

Distance from the origin of the STA to the bifurcation point. The mean distance from the origin of the STA to the bifurcation point in the MMD group (42 sides) was $73.89 \pm 15.47 \mathrm{~mm}$, and in the non-MMD group (42 sides), it was $78.71 \pm 21.68 \mathrm{~mm}$. No significant difference was present between the two groups $(\mathrm{P}=0.244$; Table VI).

\section{Discussion}

The STA has an important role in MMD, as it is the major donor vessel for the extracranial-intracranial bypass. However, whether the STA is affected in patients with MMD remained elusive. As CTA is able to clearly display the external carotid artery system (7), it was used to study the anatomical characteristics of the STA in the present study. After its origination from the external carotid artery, the STA has two characteristic vessel types (tortuous or straight) when crossing the zygomatic arch (4). In the present study, the tortuous type was indicated to be more common than the straight type in the MMD and the non-MMD groups, and there was no significant difference between the two groups. This anatomical trait has a physiological advantage, as higher tortuosity of the STA allows for more effective extension during mandibular joint movement and avoids vascular traction when opening the mouth (6).

In the present study, the diameter and trunk length of the STA were not different between the MMD and the non-MMD groups, indicating that MMD did not influence the diameter and trunk length of the STA; this result is in contrast to what occurs in the compensatory dilated tortuous middle meningeal artery in patients with MMD (8). The present results also indicated that, compared with the diameters above and below the zygomatic arch, the diameter of the STA at the midpoint of the zygomatic arch was the smallest. This difference may be a compensatory result of the movements required for chewing (9).

The diameter of the STA at the zygomatic arch midpoint was $\sim 1.7 \mathrm{~mm}$, which is generally smaller than that reported by Chen et al (10) $(2.1 \mathrm{~mm})$ in a Chinese cadaveric study. This difference may be due to the following factors: First, prior to a cadaveric study, liquid medium is always infused into the vascular system, which expands the diameters of vessels. 
Furthermore, the measured values in cadaveric studies reflect the external vascular diameter. However, the data obtained from CTA reflect the internal vascular diameter. Finally, vascular diameters measured in vivo may also be affected by systolic and diastolic blood pressure. In a Korean study based on CTA, the diameter of the STA was $\sim 1.8 \mathrm{~mm}$, which was similar to the present results (9).

The STA gives rise to the frontal branch and the parietal branch after crossing the zygomatic arch. The two branches have an important role in supplying their respective regions of the scalp. The bifurcation point is undoubtedly the core of the STA (11). In line with the method described by Kim et al (9), the posterior edge of the mandibular condyle, the upper edge of the zygomatic arch and the zygomatic process of the frontal bone as facial markers were used in the present study, making the superficial location of the STA bifurcation more accurate. The present study also indicated that the bifurcation point of the STA was closer to the posterior edge of the mandibular condyle in patients with MMD. Hence, to reduce the possibility of direct damage to the trunk of the STA during an extended pterional craniotomy in patients with MMD, it is necessary to make the incision closer to the anterior edge of the auricle.

\section{Acknowledgements}

Not applicable.

\section{Funding}

No funding was received.

\section{Availability of data and materials}

The datasets used and/or analyzed during the present study are available from the corresponding author on reasonable request.

\section{Authors' contributions}

JY and KH made substantial contributions to the conception and design of the work. KH and QL wrote the manuscript. QL, $\mathrm{KX}$ and BX collected and analyzed the data. JY and KH critically revised the manuscript. All authors read and approved the final manuscript.

\section{Ethics approval and consent to participate}

The study was approved by the Ethics Committee of the First Hospital of Jilin University (Changchun, China). Informed consent for participation in the study or use of their medical data was obtained from all participants or their legal guardians.

\section{Patient consent for publication}

Written informed consent was obtained from the patients or their guardians for publication of this manuscript and any accompanying images.

\section{Competing interests}

The authors declare that they have no competing interests.

\section{References}

1. Shin KJ, Shin HJ, Lee SH, Koh KS and Song WC: Surgical anatomy of the superficial temporal artery to prevent facial nerve injury during arterial biopsy. Clin Anat 31: 608-613, 2018.

2. Wada K, Otani N, Toyooka T, Takeuchi S, Tomiyama A and Mori K: Superficial temporal artery to anterior cerebral artery hemi-bonnet bypass using radial artery graft for prevention of complications after surgical treatment of partially thrombosed large/giant anterior cerebral artery aneurysm. J Stroke Cerebrovasc Dis 27: 3505-3510, 2018.

3. Marano SR, Fischer DW, Gaines C and Sonntag VK: Anatomical study of the superficial temporal artery. Neurosurgery 16: 786-790, 1985.

4. Yonenaga K, Tohnai I, Mitsudo K, Mori Y, Saijo H, Iwai T, Yonehara Y, Ota Y, Torigoe K and Takato T: Anatomical study of the external carotid artery and its branches for administration of superselective intra-arterial chemotherapy via the superficial temporal artery. Int J Clin Oncol 16: 654-659, 2011.

5. Li Q, Qu L, Yuan Y, Xu B, Guo Y, Xu K and Yu J: Analysis of the clinical characteristics of hemorrhagic moyamoya disease in the Jilin province of northeastern China: A single-center study of 212 cases. Biomed Rep 8: 191-197, 2018.

6. Yu J, Shi L, Guo Y, Xu B and Xu K: Progress on complications of direct bypass for moyamoya disease. Int J Med Sci 13: 578-587, 2016.

7. Korn A, Bender B, Brodoefel H, Hauser TK, Danz S, Ernemann U and Thomas $\mathrm{C}$ : Grading of carotid artery stenosis in the presence of extensive calcifications: Dual-energy CT angiography in comparison with contrast-enhanced MR angiography. Clin Neuroradiol 25: 33-40, 2015.

8. Yu J, Guo Y, Xu B and Xu K: Clinical importance of the middle meningeal artery: A review of the literature. Int J Med Sci 13: 790-799, 2016.

9. Kim BS, Jung YJ, Chang CH and Choi BY: The anatomy of the superficial temporal artery in adult koreans using 3-dimensional computed tomographic angiogram: Clinical research. J Cerebrovasc Endovasc Neurosurg 15: 145-151, 2013.

10. Chen TH, Chen CH, Shyu JF, Wu CW, Lui WY and Liu JC: Distribution of the superficial temporal artery in the Chinese adult. Plast Reconstr Surg 104: 1276-1279, 1999.

11. Aveta A, Brunetti B, Tenna S, Segreto F and Persichetti P: Superficial temporal artery perforator flap: Anatomic study of number and reliability of distal branches of the superficial temporal artery and clinical applications in three cases. Microsurgery 37: 924-929, 2017.

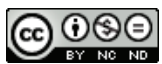

This work is licensed under a Creative Commons Attribution-NonCommercial-NoDerivatives 4.0 International (CC BY-NC-ND 4.0) License. 